\title{
Assessment of Diabetic Cardiomyopathy by Cardiovascular Magnetic Resonance T1 Mapping: Correlation with Left-Ventricular Diastolic Dysfunction and Diabetic Duration
}

\author{
Yongning Shang, ${ }^{1}$ Xiaochun Zhang, ${ }^{1}$ Weilling Leng, ${ }^{2}$ Liu Chen, ${ }^{2}$ Xiaotian Lei, ${ }^{2}$ \\ Tianjing Zhang, ${ }^{3}$ Andreas Greiser, ${ }^{4}$ Ziwen Liang, ${ }^{2}$ and Jian Wang ${ }^{1}$ \\ ${ }^{1}$ Department of Radiology, Southwest Hospital, Third Military Medical University, Chongqing, China \\ ${ }^{2}$ Department of Endocrinology, Southwest Hospital, Third Military Medical University, Chongqing, China \\ ${ }^{3}$ Northeast Asia MR Collaboration, Siemens Healthcare, Beijing, China \\ ${ }^{4}$ Siemens Healthcare, Erlangen, Germany
}

Correspondence should be addressed to Xiaochun Zhang; zxcylxyr@163.com, Ziwen Liang; ziwenliang99@163.com, and Jian Wang; wangjian_811@yahoo.com

Received 7 March 2017; Revised 9 May 2017; Accepted 28 May 2017; Published 16 July 2017

Academic Editor: Manfredi Rizzo

Copyright $\odot 2017$ Yongning Shang et al. This is an open access article distributed under the Creative Commons Attribution License, which permits unrestricted use, distribution, and reproduction in any medium, provided the original work is properly cited.

Purpose. To quantify extracellular matrix expansion with the cardiovascular magnetic resonance (CMR) T1 mapping technique and the derived extracellular volume fraction (ECV) in diabetic cardiomyopathy (DbCM) patients and to detect the relationship among ECV, duration of diabetes, and diastolic function. Materials. Thirty-eight patients with diabetic cardiomyopathy (20 males, age $54.6 \pm 8.6$ years) and thirty-two matched normal controls (15 males, age $51.4 \pm 13.6$ years) were prospectively enrolled. All of them were scanned by T1 mapping to obtain the native and postcontrast T1 values of myocardium and blood, and ECV was calculated accordingly. All patients also underwent transthoracic echocardiographic tissue Doppler imaging to assess leftventricular diastolic function. Results. There was a significant difference in ECV between the two groups (DbCMs $30.4 \pm 2.9 \%$ versus controls $27.1 \pm 2.4 \%, P<0.001)$. The duration of diabetes was positively and strongly associated with ECV $(R=0.539$, $P=0.0005)$. There was also a significant difference in ECV $(P \leq 0.001)$ among four groups $(\mathrm{A}$, controls; $\mathrm{B}$, DbCM patients with duration of diabetes $<5$ years; C, 5-10 years; and D, $>10$ years). ECV was negatively associated with LV E'/A' $(R=-0.403$, $P=0.012$ ). Conclusion. CMR T1 mapping can reflect myocardial extracellular matrix expansion in DbCM and can be a powerful technique for the early diagnosis of DbCM.

\section{Introduction}

Type 2 diabetes mellitus (T2DM), one of the most common chronic diseases globally, affects nearly four hundred million individuals [1]. Diabetic cardiomyopathy (DbCM), which is present in almost two-thirds of patients with T2DM, is defined as myocardial dysfunction occurring independently of coronary artery disease, valvular dysfunction, or hypertension $[2,3]$.

The minimal criteria for the diagnosis of $\mathrm{DbCM}$ include the presence of left-ventricular (LV) diastolic dysfunction but preserved systolic function, which makes early diagnosis of DbCM difficult. Diffuse expression, accumulation, and crosslinking of interstitial collagen can expand the extracellular space of the myocardium, which contributes to the development and progression of DbCM [4]. Pathologic biopsy is a gold standard for assessing myocardial fibrosis. Whereas many techniques have been developed to assess myocardial fibrosis (such as CT, echocardiography, SPECT, and PET), these are less accurate than contrast-enhanced cardiovascular magnetic resonance (CMR) [5]. The conventional CMR late gadolinium enhancement (LGE) method, the validation, and prognostic advantages of which have been examined extensively in a variety of pathologic studies have been 
described as a superior tool to other imaging modalities in the identification of cardiac scars or local fibrosis [5]. Despite adequate contrast between "normal" myocardium and scar tissue, diffuse and interstitial fibrosis in many disease processes cannot be effectively detected by LGE. However, the elevated sensitivity of T1 mapping has proven useful in these cases $[5,6]$.

Novel cardiac MR T1 mapping techniques make it feasible to measure myocardial extracellular matrix (ECM) expansion in vivo. Extracellular volume fraction (ECV), derived from native-T1 mapping and postcontrast T1 mapping after the administration of a bolus of gadolinium (Gd) contrast, can quantify myocardial ECM expansion. ECV quantification correlates well with histology [7] and is highly reproducible [8] and sensitive [9]. For those reasons, ECV has been regarded as an essential biomarker for quantifying the full spectrum of diffuse myocardial fibrosis. In the $\mathrm{DbCM}$ setting, the relationships among mapping parameters (including native T1 value, postcontrast T1 value, and ECV) and the duration of diabetes and diastolic function should be elucidated.

The specific purpose of the present study was to quantify ECM expansion with the cardiac MR T1 mapping technique and the derived ECV in $\mathrm{DbCM}$, detect the relationship between ECV and the duration of diabetes, and evaluate the relationship between ECV and diastolic function.

\section{Materials and Methods}

2.1. Study Patients. This study was approved by the Institutional Review Board (IRB) of our hospital, and all subjects gave informed consent. This study was conducted in our hospital between June 2015 and September 2016. The inclusion criteria for DbCM patients included (1) an initial diagnosis of T2DM according to the criteria of the World Health Organization [10]; (2) left-ventricular early diastolic dysfunction (echocardiographic Doppler Tissue Imaging $E^{\prime} / A^{\prime}<1$, derived from the ratio of early to late diastolic mitral annulus velocity [11]); (3) no history of symptomatic coronary heart disease (no clinical manifestation and no abnormal signal on first pass perfusion and late gadolinium enhancement images); and (4) no hypertension. Exclusion criteria included glomerular filtration rate (GFR) $\leq 30 \mathrm{~mL} /$ $\min / 1.7 \mathrm{~m}^{2}$ and other standard contraindications to cardiac MR. Forty consecutive participants were recruited for cardiac MR examination. Two participants were excluded because of the presence of high heart rate ( $>100$ beats/min) during physical examination. Thirty-two normal controls were recruited from the community population during the same period.

2.2. Anthropometry and Biochemistry Exams. All DbCM patients and normal controls underwent height, weight, and blood pressure examinations. Blood samples were collected at about 30 minutes before scanning. Those blood samples were immediately sent to the Department of Clinical Laboratory and Nuclear Medicine and immediately

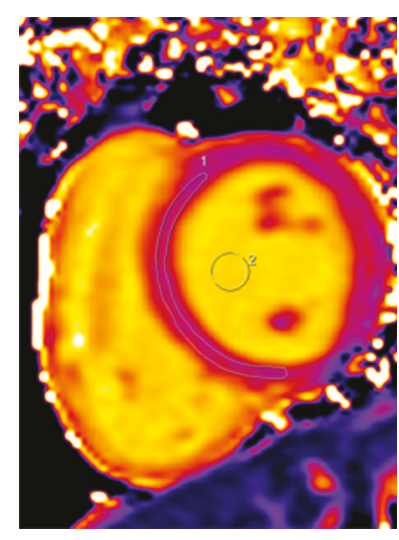

(a)

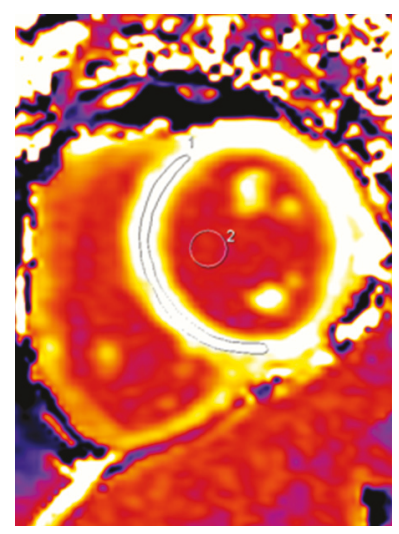

(b)
Figure 1: ROIs on native (a) and postcontrast (b) T1 mapping.

analyzed to obtain haematocrit (HCT), serum glucose, glycated hemoglobin ( $\mathrm{HbAlc})$, insulin, C-peptide, creatinine, cystatin C, triglycerides (TG), total cholesterol (TC), highdensity lipoprotein cholesterol (HDL) and low-density lipoprotein cholesterol (LDL) levels, myocardium zymogram examination, and markers of myocardial damage.

2.3. Echocardiography. All patients underwent transthoracic echocardiography at rest using a commercially available ultrasound machine and transducer (Philips IE33 color Doppler scanner, S5-1 heart probe, the Netherlands). According to the guidelines of the American Society of Echocardiography [11], tissue Doppler imaging (TDI) was used to measure left-ventricular diastolic function. The sample volume was placed at the mitral valve annulus in an apical four-chamber view. The septal mitral valve annulus early E' wave (passive left-ventricular filling) and late A' wave (atrial contraction) velocity and the ratio of the two (E'/A') were assessed.

2.4. Cardiac MR Protocol. All DbCM patients and normal controls underwent cardiac MR examination on a 3T system (MAGNETOM Trio Tim, Siemens Healthcare, Erlangen, Germany). They were placed in a supine position using a 6 -channel body matrix plus 2 rows of the spine array. Breath holding after expiration and electrocardiographic gating were used when necessary. LGE was performed about $15 \mathrm{~min}$ after bolus injection of $0.2 \mathrm{mmol} / \mathrm{kg}$ i.v. gadoteric acid meglumine bolus (Dotarem, Guerbet, BP7400, F95943, Roissy CdG Cedex, France) with the Phase Sensitive Inversion Recovery sequence (TR/TE 680/1.94 ms, slice thickness $8 \mathrm{~mm}$, spacing between slices $1.6 \mathrm{~mm}$ ) to exclude local myocardial fibrosis.

2.5. T1 Mapping. T1 mapping was performed using a modified Look-Locker Inversion Recovery (MOLLI) prototype sequence (Siemens Work-in-Progress no. 448B, (VB17A)) [12] (FOV $400 \times 300 \mathrm{~mm}^{2}$, matrix $256 \times 166$, $6 \mathrm{~mm}$ thickness, TR/TE $301.7 / 1.09 \mathrm{~ms}$, flip angle 35 degrees, 6/8 partial-Fourier $k$-space sampling, PAT factor 2). Spacing in between inversion experiments and image 
TABLE 1: Anthropometry and biochemical characteristics.

\begin{tabular}{|c|c|c|c|}
\hline & $\operatorname{DbCM}(N=38)$ & Control $(N=32)$ & $P$ value \\
\hline \multicolumn{4}{|l|}{ Anthropometry } \\
\hline Age, y & $54.6 \pm 8.6$ & $51.4 \pm 13.6$ & 0.229 \\
\hline Diabetic history, y & $7.0[2.8-11.0]$ & & \\
\hline Male, $n(\%)$ & $20(52.6)$ & $15(46.9)$ & 0.405 \\
\hline Height, m & $1.62 \pm 0.07$ & $1.61 \pm 0.07$ & 0.711 \\
\hline Weight, kg & $63.9 \pm 9.8$ & $61.3 \pm 10.5$ & 0.296 \\
\hline Body mass index, $\mathrm{kg} / \mathrm{m}^{2}$ & $24.3 \pm 2.7$ & $23.5 \pm 3.1$ & 0.248 \\
\hline Body surface area, $\mathrm{m}^{2}$ & $1.68 \pm 0.15$ & $1.65 \pm 0.17$ & 0.491 \\
\hline Systolic blood pressure, $\mathrm{mmHg}$ & $118.5 \pm 10.5$ & $119.0 \pm 11.4$ & 0.867 \\
\hline Diastolic blood pressure, $\mathrm{mmHg}$ & $80.9 \pm 5.9$ & $83.4 \pm 7.6$ & 0.118 \\
\hline \multicolumn{4}{|l|}{ Biochemical exam } \\
\hline Urine microalbumin, $\mathrm{mg} / \mathrm{dL}$ & $0.3[0.1-3.0]$ & $0.9[0.6-1.7]$ & 0.072 \\
\hline Blood urea nitrogen, $\mathrm{mmol} / \mathrm{L}$ & $5.8[4.8-7.0]$ & $5.8[5.1-6.8]$ & 0.810 \\
\hline Creatinine, $\mu \mathrm{mol} / \mathrm{L}$ & $60.0[54.6-71.6]$ & $64.0[57.3-76.3]$ & 0.632 \\
\hline Cystatin C, mg/L & $0.81 \pm 0.20$ & $0.76 \pm 0.16$ & 0.256 \\
\hline AST, IU/L & $29.3[23.2-35.4]$ & $20.2[17.3-27.6]$ & 0.001 \\
\hline LDH, IU/L & $188.1 \pm 54.0$ & $196.4 \pm 46.0$ & 0.549 \\
\hline$\alpha-\mathrm{HBDH}, \mathrm{IU} / \mathrm{L}$ & $126.0 \pm 28.7$ & $131.0 \pm 37.9$ & 0.581 \\
\hline $\mathrm{CK}, \mathrm{IU} / \mathrm{L}$ & $93.7[62.2-130.5]$ & $88.5[66.2-105.2]$ & 0.576 \\
\hline Ischemia modified albumin, $\mathrm{U} / \mathrm{mL}$ & $82.6 \pm 7.7$ & $73.6 \pm 5.7$ & $\leq \mathbf{0 . 0 0 1}$ \\
\hline Total cholesterol, mmol/L & $5.4[4.0-6.3]$ & $5.2[4.8-5.9]$ & 0.926 \\
\hline Triglycerides, mmol/L & $2.1[1.1-3.6]$ & $1.4[0.9-2.0]$ & 0.013 \\
\hline $\mathrm{HDL}, \mathrm{mmol} / \mathrm{L}$ & $1.2[1.0-1.3]$ & $1.4[1.2-1.7]$ & 0.001 \\
\hline $\mathrm{LDL}, \mathrm{mmol} / \mathrm{L}$ & $3.4[2.5-3.9]$ & $3.3[3.1-3.7]$ & 0.440 \\
\hline Glucose, $\mathrm{mmol} / \mathrm{L}$ & $8.2[6.5-10.6]$ & $5.3[4.8-5.8]$ & $\leq 0.001$ \\
\hline Glycated hemoglobin, \% & $7.4[6.7-8.9]$ & $5.6[5.4-5.8]$ & $\leq \mathbf{0 . 0 0 1}$ \\
\hline Troponin, $10^{-3} \mu \mathrm{g} / \mathrm{L}$ & $8[5-12]$ & $5[3-6]$ & 0.001 \\
\hline Myoglobin, ng/mL & $26.5[21.0-41.3]$ & $30.4[22.0-38.5]$ & 0.636 \\
\hline Insulin, $\mu \mathrm{IU} / \mathrm{mL}$ & $18.5 \pm 9.8$ & $12.8 \pm 4.5$ & 0.021 \\
\hline C peptide, $\mathrm{ng} / \mathrm{mL}$ & $1.8[1.3-2.6]$ & $1.1[0.8-1.8]$ & 0.016 \\
\hline Haematocrit, \% & $39.8 \pm 4.4$ & $41.8 \pm 4.5$ & 0.055 \\
\hline \multicolumn{4}{|l|}{ Medications } \\
\hline Angiotensin-converting enzyme inhibitors, $n(\%)$ & $5(13.2)$ & & \\
\hline Statin, $n(\%)$ & $26(68.4)$ & & \\
\hline Aspirin, $n(\%)$ & $4(10.5)$ & & \\
\hline \multicolumn{4}{|l|}{ Echocardiography } \\
\hline Doppler mitral annulus velocity E'/A' & $0.78 \pm 0.09$ & & \\
\hline
\end{tabular}

AST: aspartate aminotransferase; LDH: lactate dehydrogenase; $\alpha$-HBDH: alpha-hydroxybutyrate dehydrogenase; CK: creatine kinase; HDL: high-density lipoprotein cholesterol; LDL: low-density lipoprotein cholesterol.

acquisition were both heartbeat-based (precontrast T1 recovery: a 5 (4) 2 sampling scheme, trigger delay 80 msec; postcontrast T1 recovery: a 4 (1) 2 (2) 2 sampling scheme, trigger delay $160 \mathrm{msec})$. Native T1 mapping was performed at basal, middle, and apical short axis of leftventricular slices. Postcontrast T1 mapping was performed $20 \mathrm{~min}$ after bolus injection at the identical slice positions. T1 maps were generated online immediately after the scan. The motion correction (MoCo) technique was used.

\subsection{Cardiac MR Analysis}

2.6.1. T1 Mapping. T1 mapping images were analyzed by two blinded radiologists (observer 1, 12 years of experience; observer 2, 10 years of experience) with a multimodality processing platform (syngo MR, Siemens Healthcare, Erlangen, Germany). The region of interest (ROI) was selected manually on the middle third of interventricular septum myocardium to avoid epicardial and endocardial partial-volume effects. The other ROI in the left-ventricular 
blood pool was located far away from both papillary muscles and myocardium to avoid partial-volume effects (Figure 1). The ECV of each slice was calculated with the following formula [13]:

$\mathrm{ECV}=(1-$ haematocrit $) \frac{(1 / \mathrm{T} 1 \text { myo post })-(1 / \mathrm{T} 1 \text { myo native })}{(1 / \mathrm{T} 1 \text { blood post })-(1 / \mathrm{T} 1 \text { blood native })}$,

where "myo" means myocardium. We averaged ECV measures of basal, middle, and apical short-axis images to obtain the final ECV value.

2.7. Statistical Analysis. Categorical variables were presented as percentages. Continuous variables were summarized as the mean and standard deviation (SD). Independent $t$-tests were used to evaluate the differences between the DbCM group and the normal control group; the Mann-Whitney $U$ test was performed when standard variance was heterogeneous. One-way ANOVA was performed to test the differences among the four groups, and the Bonferroni test was used as a post hoc test. The Kolmogorov-Smirnov test was used as a normality test. The relationships between bivariate were analyzed using Pearson's or Spearman's method. Statistical tests were two-tailed, and $P<0.05$ was considered statistically significant. Statistical analysis was performed using commercially available software (SPSS for Windows 21.0, Inc, Chicago, IL, USA).

\section{Results}

The results of anthropometry and biochemistry assessments are shown in Table 1.

Thirty-eight patients (20 males, age $54.6 \pm 8.6$ years, the duration of diabetes 7.0 [2.8-11.0] years) with DbCM and thirty-two normal controls ( 15 males, age $51.4 \pm 13.6$ years) were studied. Fasting blood glucose and $\mathrm{HbA1c}$ were higher in patients with DbCM than in normal controls. DbCM was also associated with higher TG, HDL, aspartate aminotransferase (AST), ischemia modified albumin (IMA), and troponin levels. There were no significant differences between the two groups in age, gender, height, weight, and so on (all $P>0.05$ ).

The results about standard cardiac MR parameters on morphology (LV end diastolic volume, end systolic volume, and mass) and systolic function have been published in our previous paper [14]. There were no visually observed abnormal signal on LGE images of all participants, suggesting no local myocardial scar.

DbCM patients and controls had similar myocardial native $\mathrm{T} 1$ values (DbCMs $1213.5 \pm 57.5 \mathrm{~ms}$ versus controls $1212.8 \pm 41.4 \mathrm{~ms}, P=0.950)$ and postcontrast T1 values (DbCMs $518.8 \pm 45.1 \mathrm{~ms}$ versus controls $528.9 \pm 38.9 \mathrm{~ms}$, $P=0.324)$. However, there was a significant difference in ECV between the two groups (DbCMs $30.4 \pm 2.9 \%$ versus controls $27.1 \pm 2.4 \%, P<0.001$, Figure 2), indicating the presence of extracellular volume expansion in the myocardium of patients with DbCM.

Table 2 shows Pearson's correlation and Spearman's correlation analysis of ECV to biochemical characteristics,

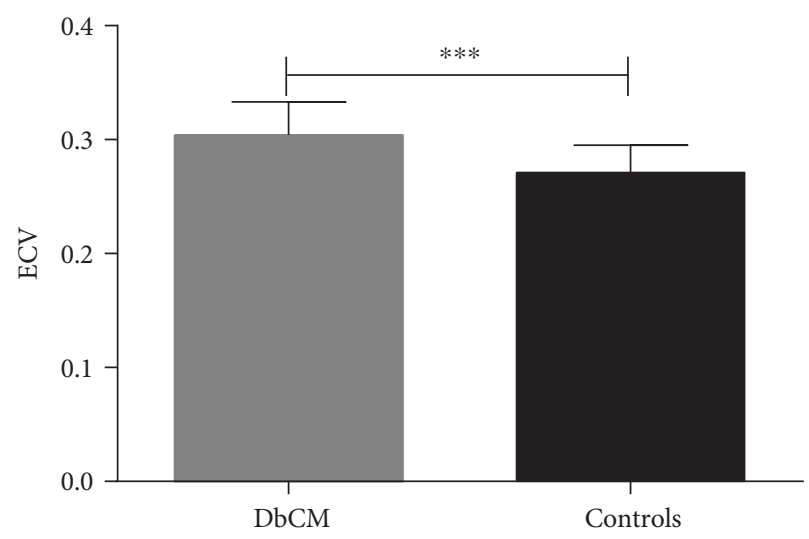

FIgURE 2: Differences in left-ventricular ECV between patients with DbCM and control subjects. $* * *$ means $p<0.001$.

which are typically different in DbCM. ECV correlated significantly with insulin.

Pearson's correlation analysis showed that ECV was positively associated with the duration of diabetes $(R=0.539$, $P=0.0005$; Figure 3 ), and multivariable stepwise linear regression indicated that the duration of diabetes was an independent and strong predictor of ECV (Table 3). Native T1 value was positively associated with the duration of diabetes in univariable correlation analysis $(R=0.439, P=0.006$, Figure $4(\mathrm{a})$ ) but not in multivariable correlation analysis when adjusted for insulin, age, height, weight, BMI, BSA, systolic blood pressure, and diastolic blood pressure $(R=0.157$, $P=0.548)$. Postcontrast T1 value did not correlate with the duration of diabetes in uni- and multivariable correlation analyses $(R=-0.179, P=0.282 ; R=0.211, P=0.415$, respectively, Figure 4(b)).

Based on the duration of diabetes (7.0 [2.8-11.0] years), all $\mathrm{DbCM}$ patients were divided into three groups (group B, $<5$ years; group C, 5-10 years; and group D, >10 years). There was a significant difference in ECV (ANOVA $P \leq 0.001$, Table 4) among controls (group A) and groups B, C, and D. Nevertheless, native and postcontrast myocardial T1 values were not significantly different among four groups ( $P=0.086$ and $P=0.596$, resp., Table 4$)$.

All patients with DbCM exhibited decreased echocardiographic TDI mitral annulus velocity E'/A' (all E'/ $\left.\mathrm{A}^{\prime}<1\right)$, and Pearson's correlation analysis showed that ECV was negatively associated with E'/A' $(R=-0.403$, $P=0.012$; Figure 5). E'/A' also correlated with native T1 value $(R=-0.424, P=0.008$, Figure $4(\mathrm{c}))$ and postcontrast T1 $(R=0.328, P=0.045$, Figure $4(\mathrm{~d}))$. We previously showed that there was no significant difference in EF between DbCM patients and controls [14]. Here, we found that EF did not correlate with ECV in DbCM patients $(R=-0.023, P=0.892)$.

\section{Discussion}

DbCM is defined as myocardial dysfunction that occurs independently of coronary artery disease, valvular dysfunction, or hypertension $[2,3]$. DbCM is associated with altered 
TABLE 2: Relationship between ECV and biochemical characteristics.

\begin{tabular}{lccccccrrr}
\hline & AST & TG & HDL & Glu & HbA1c & Troponin & C-peptide & IMA & Insulin \\
\hline $\begin{array}{l}\text { Rho } \\
R\end{array}$ & 0.077 & -0.116 & -0.135 & -0.138 & 0.025 & -0.341 & -0.108 & & 0.486 \\
$P$ & & & & & & & 0.239 & 0.600 & 0.250 \\
\hline
\end{tabular}

Rho: Spearman's correlation analyzed for nonnormal distributions. $R$ : Pearson's correlation analyzed for normal distributions; AST: aspartate aminotransferase; TG: triglycerides; HDL: high-density lipoprotein cholesterol; Glu: glucose; HbA1c: glycated hemoglobin; IMA: ischemia modified albumin.

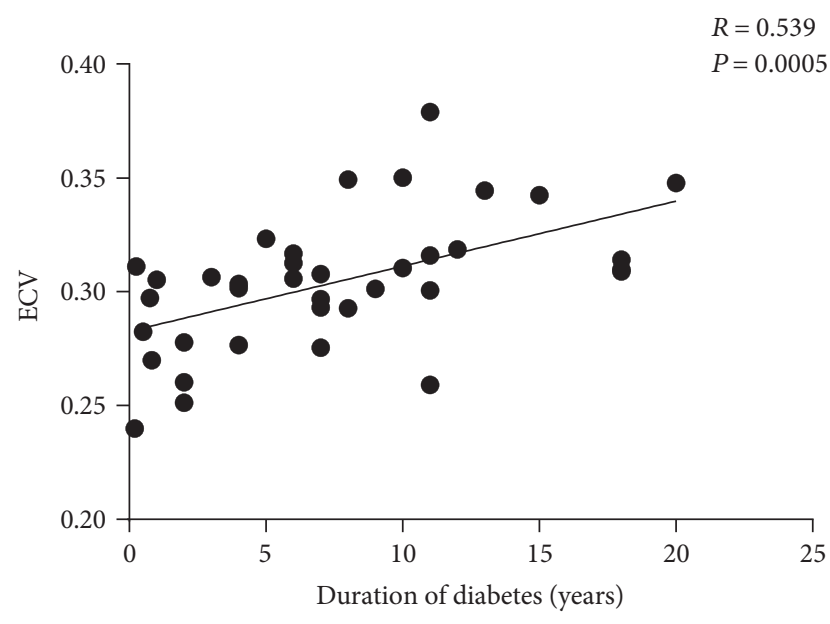

Figure 3: Relationship between ECV and the duration of diabetes.

left-ventricular geometry, function, and tissue characterization $[13,15,16]$. Using cardiac MR, we found that (1) DbCM is accompanied by increased ECV, which indicates extracellular volume expansion; (2) ECV is associated with the duration of diabetes; and (3) increased ECV correlates with reduced TDI E'/A'.

Although native $\mathrm{T} 1$ and postcontrast $\mathrm{T} 1 \mathrm{did}$ not statistically differ between DMs and controls, there was a trend that native $\mathrm{T} 1$ was longer and postcontrast $\mathrm{T} 1$ was shorter in DMs. As we know, ECV was calculated as $(1-\mathrm{HCT}) *$ (1/(T1 myo post $)-1 /(\mathrm{T} 1$ myo native $)) /(1 /(\mathrm{T} 1$ blood post $)-$ $1 /(\mathrm{T} 1$ blood native)). Combination of the differences of native $\mathrm{T} 1$ and post contrast $\mathrm{T} 1$ may lead to statistical difference in ECV. It demonstrated that ECV may be more sensitive than native and postcontrast T1 to indicate DM-related myocardial change. This might have significant clinical implications for diseases that are characterized by an alteration in ECV, such as DbCM. With increased sensitivity, the disease could potentially be detected earlier when only subtle differences in ECV may be present.

Several studies have shown that DbCM is associated with increased collagen, especially type III collagen, in the myocardial interstitium of humans [17] and animal models [18]. However, previous studies have reported controversial results about the relationship between DbCM and ECM expansion $[19,20]$. ECV, derived from T1 mapping cardiac $\mathrm{MR}$, is considered as the biomarker of cardiac fibrosis, which is caused by the deposition of perivascular and intermyofibrillar collagen [21]. DbCM is expected to be associated with increased ECV. However, previous studies presented conflicting results on the elevation of ECV in patients with
TABLE 3: Uni- and multivariable correlation analyses between ECV and the duration of diabetes.

\begin{tabular}{lcc}
\hline Variable & $R$ & $P$ value \\
\hline The duration of diabetes & 0.539 & $\leq \mathbf{0 . 0 0 1}$ \\
$\begin{array}{l}\text { The duration of diabetes, insulin } \\
\begin{array}{l}\text { The duration of diabetes, insulin, age, } \\
\text { height, weight, BMI, BSA, systolic } \\
\text { blood pressure, and diastolic blood pressure }\end{array}\end{array}$ & 0.464 & $\mathbf{0 . 0 2 2}$ \\
\hline
\end{tabular}

T2DM or DbCM. Khan et al. [16] and Levelt et al. [15] reported that patients and control subjects had similar ECVs, but the patient population in the former study consisted of young adults, and the patient population of the latter study was too highly selected (no diabetic complications or Hba1C $>9 \%)$. Wong et al. [13] indicated that diabetes (accompanied by $85 \%$ hypertension, 33\% prior coronary revascularization, and $11 \%$ prior acute myocardial infarction) was associated with higher ECVs than nondiabetes. Other factors, such as hypertension, may influence the study results. Therefore, in the current study, we carefully selected patients and normal control subjects. We found that $\mathrm{DbCM}$ was associated with elevated ECV, despite the fact that patients with DbCM had similar native and postcontrast T1 values as healthy control subjects, which was consistent with a previous study [16]. This may indicate the presence of diffuse myocardial fibrosis in patients with DbCM.

Interestingly, we found that duration of diabetes, not hyperglycemia, HbAlc, troponin, or IMA, was an independent predictor of ECV and group with the longer duration of diabetes was associated with higher ECV value. Recent studies showed that hyperglycemia, even transient hyperglycemia (a few hours), can cause persistent (a few days) damage to cells by disrupting the signal feedback loop [22] and hyperglycemia can sufficiently promote the proliferation of cardiac fibroblast [23]. HbAlc is a marker of time-averaged glucose level. Neither glucose nor HbAlc can reveal the severity of long-term damage to the myocardium. Elevated troponin and IMA may indicate an impairment of cardiac myocytes, but these markers cannot reflect the severity of long-term damage. The relationship between diabetic history and ECV indicates that ECV may represent the long-term effects of DbCM on the myocardium. This should be confirmed by further studies with more participants.

Our results show that there is a significant relationship between impaired LV diastolic function and increased ECV in patients with $\mathrm{DbCM}$, although the association between 


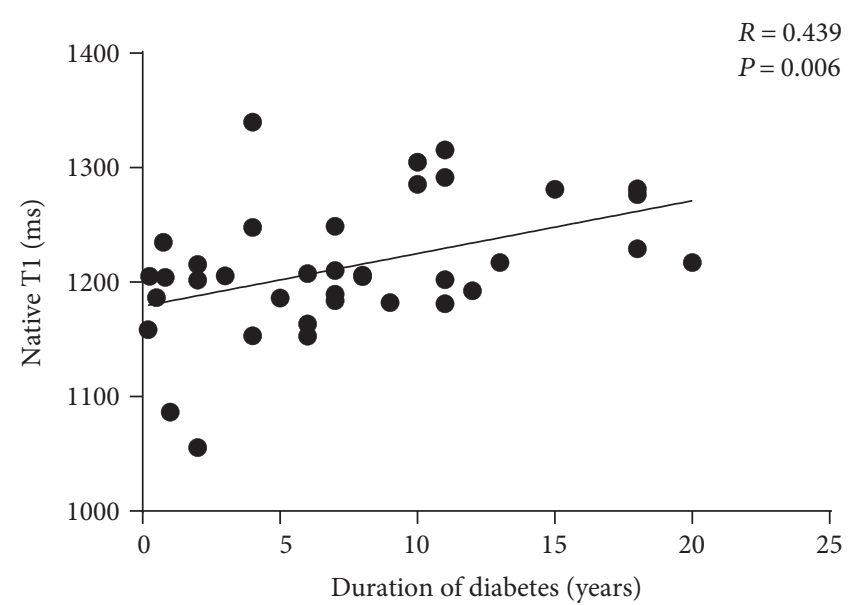

(a)

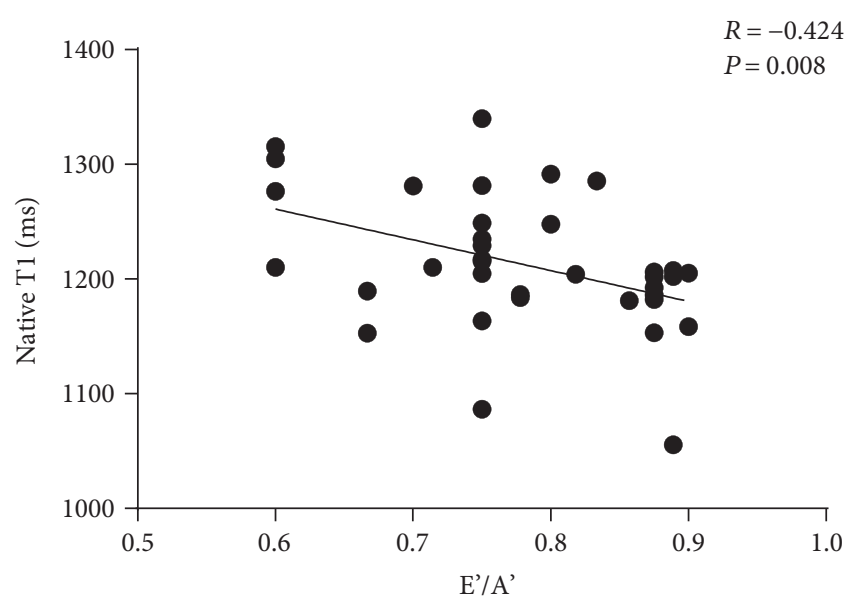

(c)

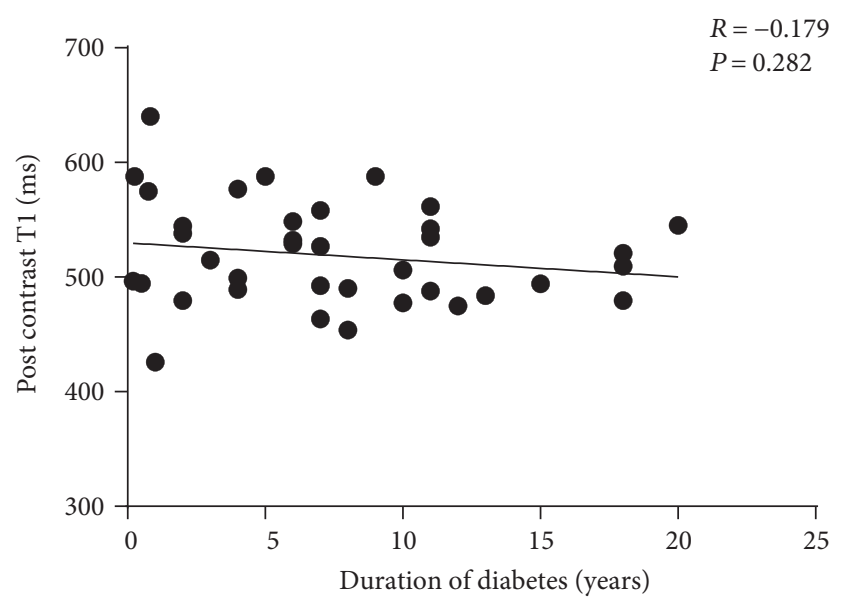

(b)

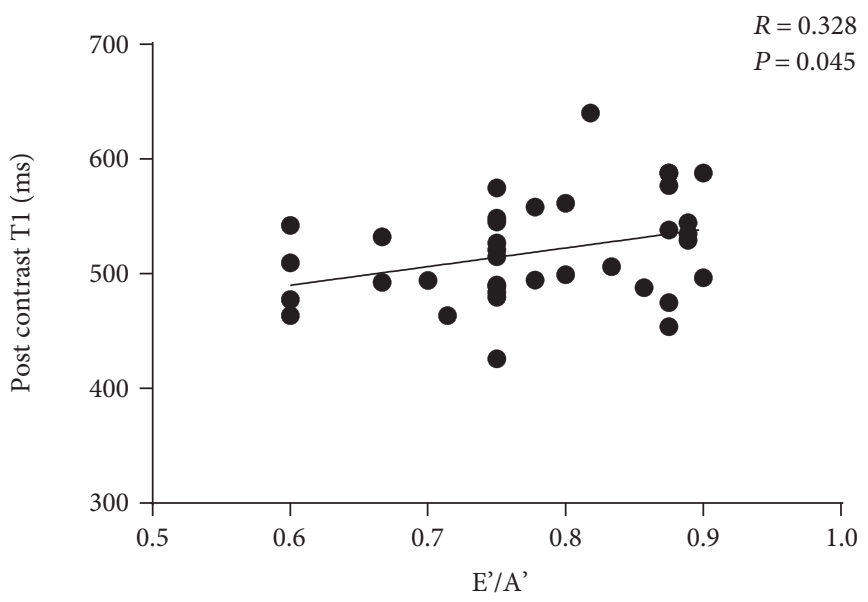

(d)

Figure 4: Correlations among myocardial T1 value, left-ventricular TDI E'/A', and duration of diabetes. (a) Correlation between native myocardial T1 value and duration of diabetes. (b) Correlation between postcontrast myocardial T1 value and duration of diabetes. (c) Correlation between native myocardial T1 value and TDI E'/A'. (d) Correlation between postcontrast myocardial T1 value and TDI E'/A'.

TABLE 4: Left-ventricular myocardial T1 value and extracellular volume in controls and DbCM patients.

\begin{tabular}{|c|c|c|c|c|c|}
\hline & $\begin{array}{c}\text { Controls } \\
(\mathrm{A}, n=32)\end{array}$ & $\begin{array}{c}<5 \text { years } \\
(\mathrm{B}, n=13)\end{array}$ & $\begin{array}{l}5-10 \text { years } \\
(\mathrm{C}, n=14)\end{array}$ & $\begin{array}{l}>10 \text { years } \\
(\mathrm{D}, n=11)\end{array}$ & $P$ value \\
\hline Native myocardial T1 value, $\mathrm{ms}$ & $1212.8 \pm 41.4$ & $1191.9 \pm 71.0$ & $1209.7 \pm 43.1$ & $1244.1 \pm 46.1$ & 0.086 \\
\hline Postcontrast myocardial T1 value, ms & $528.9 \pm 38.9$ & $527.8 \pm 56.7$ & $515.5 \pm 44.6$ & $512.2 \pm 30.3$ & 0.596 \\
\hline Extracellular volume fraction, $\%$ & $27.1 \pm 2.4$ & $28.3 \pm 2.3$ & $30.9 \pm 2.1$ & $32.2 \pm 3.1$ & $\leq 0.001$ \\
\hline
\end{tabular}

ECV and diastolic dysfunction has been reported in patients with heart failure with preserved ejection fraction [24] and in children and young adults with congenital aortic stenosis [25]. The findings concerning the relationship between LV function and fibrosis are in agreement with those of previous studies. On the one hand, elevated collagen content in the myocardium affects cardiac relaxation. Using a rat model, Mizushige et al. [26] showed that diabetes was associated with low transmitral inflow velocity of early filling rate, high late filling rate, and the severity of the impairment becoming worse with disease progression. Elevated myocardial collagen content is closely associated with impaired left-ventricular diastolic function [23]. On the other hand, the formation of advanced glycosylation end products on the myocardium induces collagen molecules in situ to cross-link with each other, which leads to the impaired elasticity of collagen and a subsequent decrease in myocardial compliance [27]. Increased myocardial stiffness along with diabetes progression induces impaired LV diastolic function.

There are some limitations in this preliminary study. First, the overall number of subjects was limited; thus, the inhomogeneity of the patient group may have introduced bias. Second, we did not recruit patients with a GFR $\leq 30 \mathrm{~mL} / \mathrm{min} / 1.7 \mathrm{~m}^{2}$, and the patients in the study 


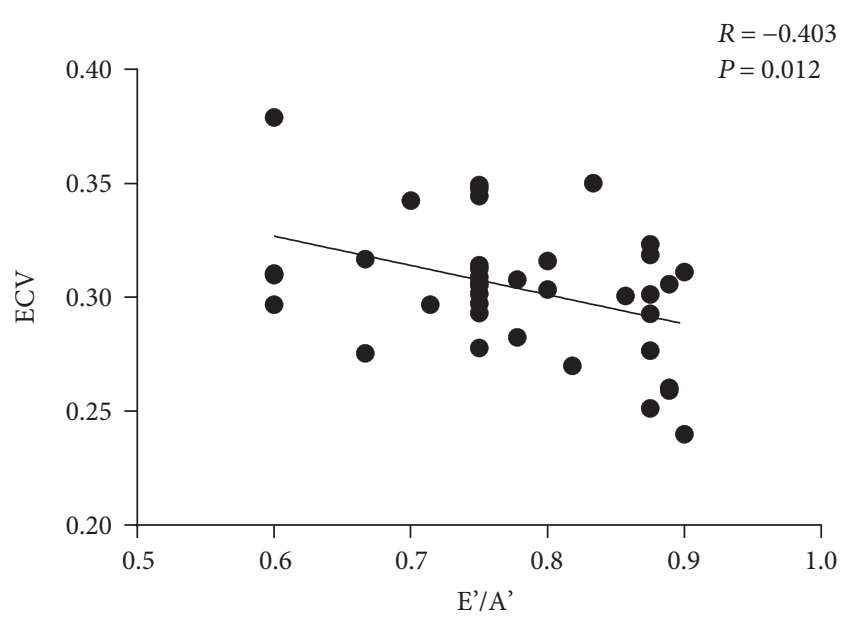

FIgURE 5: Relationship between ECV and left-ventricular TDI E'/A'.

received different treatments. Finally, a myocardium zymogram examination was performed, markers of myocardial damage were assessed in only $84 \%$ of the patients and controls, and urine microalbumin was examined in only $74 \%$ of individuals.

\section{Conclusion}

When compared to normal control participants matched to age, BMI, and BSA, patients with DbCM exhibited elevated ECV detected using cardiovascular magnetic resonance T1 mapping technique. LV ECV correlates well with the duration of diabetes and LV impaired diastolic function. Cardiovascular magnetic resonance T1 mapping can reflect myocardial extracellular matrix expansion in patients with diabetic cardiomyopathy and may be a powerful technique for early diagnosis of diabetic cardiomyopathy.

\section{Conflicts of Interest}

The authors declare that they have no competing interests.

\section{Acknowledgments}

This work was supported by the National Natural Science Foundation of China (Grant nos. 81471647, 81571748).

\section{References}

[1] J. M. Pappachan, G. I. Varughese, R. Sriraman, and G. Arunagirinathan, "Diabetic cardiomyopathy: pathophysiology, diagnostic evaluation and management," World Journal of Diabetes, vol. 4, no. 5, pp. 177-189, 2013.

[2] S. Boudina and E. D. Abel, "Diabetic cardiomyopathy revisited," Circulation, vol. 115, no. 25, pp. 3213-3223, 2007.

[3] M. N. Tillquist and T. M. Maddox, "Update on diabetic cardiomyopathy: inches forward, miles to go," Current Diabetes Reports, vol. 12, no. 3, pp. 305-313, 2012.

[4] G. Jia, V. G. DeMarco, and J. R. Sowers, "Insulin resistance and hyperinsulinaemia in diabetic cardiomyopathy," Nature Reviews. Endocrinology, vol. 12, no. 3, pp. 144-153, 2016.
[5] B. Ambale-Venkatesh and J. A. Lima, "Cardiac MRI: a central prognostic tool in myocardial fibrosis," Nature Reviews. Cardiology, vol. 12, no. 1, pp. 18-29, 2015.

[6] V. O. Puntmann, E. Peker, Y. Chandrashekhar, and E. Nagel, "T1 mapping in characterizing myocardial disease: a comprehensive review," Circulation Research, vol. 119, no. 2, pp. 277-299, 2016.

[7] M. Fontana, S. K. White, S. M. Banypersad et al., "Comparison of T1 mapping techniques for ECV quantification. Histological validation and reproducibility of ShMOLLI versus multibreath-hold T1 quantification equilibrium contrast CMR," Journal of Cardiovascular Magnetic Resonance, vol. 14, p. 88, 2012.

[8] N. Kawel, M. Nacif, A. Zavodni et al., "T1 mapping of the myocardium: intra-individual assessment of the effect of field strength, cardiac cycle and variation by myocardial region," Journal of Cardiovascular Magnetic Resonance, vol. 14, p. 27, 2012.

[9] P. Kellman, J. R. Wilson, H. Xue et al., "Extracellular volume fraction mapping in the myocardium, part 2: initial clinical experience," Journal of Cardiovascular Magnetic Resonance, vol. 14, p. 64, 2012.

[10] K. G. Alberti and P. Z. Zimmet, "Definition, diagnosis and classification of diabetes mellitus and its complications. Part 1: diagnosis and classification of diabetes mellitus provisional report of a WHO consultation," Diabetic Medicine, vol. 15, no. 7, pp. 539-553, 1998.

[11] S. F. Nagueh, C. P. Appleton, T. C. Gillebert et al., "Recommendations for the evaluation of left ventricular diastolic function by echocardiography," Journal of the American Society of Echocardiography, vol. 22, no. 2, pp. 107-133, 2009.

[12] L. Zhao, S. Li, X. Ma et al., "Systolic MOLLI T1 mapping with heart-rate-dependent pulse sequence sampling scheme is feasible in patients with atrial fibrillation," Journal of Cardiovascular Magnetic Resonance, vol. 18, p. 13, 2016.

[13] T. C. Wong, K. M. Piehler, I. A. Kang et al., "Myocardial extracellular volume fraction quantified by cardiovascular magnetic resonance is increased in diabetes and associated with mortality and incident heart failure admission," European Heart Journal, vol. 35, no. 10, pp. 657-664, 2014.

[14] Y. Shang, X. Zhang, L. Chen et al., "Assessment of left ventricular structural remodelling in patients with diabetic cardiomyopathy by cardiovascular magnetic resonance," Journal of Diabetes Research, vol. 2016, Article ID 4786925, 8 pages, 2016.

[15] E. Levelt, M. Mahmod, S. K. Piechnik et al., "Relationship between left ventricular structural and metabolic remodeling in type 2 diabetes," Diabetes, vol. 65, no. 1, pp. 44-52, 2016.

[16] J. N. Khan, E. G. Wilmot, M. Leggate et al., "Subclinical diastolic dysfunction in young adults with type 2 diabetes mellitus: a multiparametric contrast-enhanced cardiovascular magnetic resonance pilot study assessing potential mechanisms," European Heart Journal. Cardiovascular Imaging, vol. 15, no. 11, pp. 1263-1269, 2014.

[17] H. Bugger and E. D. Abel, "Molecular mechanisms of diabetic cardiomyopathy," Diabetologia, vol. 57, no. 4, pp. 660-671, 2014.

[18] V. P. Singh, B. Le, R. Khode, K. M. Baker, and R. Kumar, "Intracellular angiotensin II production in diabetic rats is correlated with cardiomyocyte apoptosis, oxidative stress, and cardiac fibrosis," Diabetes, vol. 57, no. 12, pp. 32973306, 2008. 
[19] A. C. Ng, D. Auger, V. Delgado et al., "Association between diffuse myocardial fibrosis by cardiac magnetic resonance contrast-enhanced $\mathrm{T}(1)$ mapping and subclinical myocardial dysfunction in diabetic patients: a pilot study," Circulation. Cardiovascular Imaging, vol. 5, no. 1, pp. 51-59, 2012.

[20] M. Ugander, A. J. Oki, L. Y. Hsu et al., "Extracellular volume imaging by magnetic resonance imaging provides insights into overt and sub-clinical myocardial pathology," European Heart Journal, vol. 33, no. 10, pp. 1268-1278, 2012.

[21] E. B. Schelbert and D. R. Messroghli, "State of the art: clinical applications of cardiac T1 mapping," Radiology, vol. 278, no. 3, pp. 658-676, 2016.

[22] F. Giacco, X. Du, A. Carratu et al., "GLP-1 cleavage product reverses persistent ROS generation after transient hyperglycemia by disrupting an ROS-generating feedback loop," Diabetes, vol. 64, no. 9, pp. 3273-3284, 2015.

[23] K. Huynh, B. C. Bernardo, J. R. McMullen, and R. H. Ritchie, "Diabetic cardiomyopathy: mechanisms and new treatment strategies targeting antioxidant signaling pathways," Pharmacology \& Therapeutics, vol. 142, no. 3, pp. 375-415, 2014.

[24] M. Y. Su, L. Y. Lin, Y. H. Tseng et al., "CMR-verified diffuse myocardial fibrosis is associated with diastolic dysfunction in HFpEF," JACC. Cardiovascular Imaging, vol. 7, no. 10, pp. 991-997, 2014.

[25] S. M. Dusenbery, M. Jerosch-Herold, C. Rickers et al., "Myocardial extracellular remodeling is associated with ventricular diastolic dysfunction in children and young adults with congenital aortic stenosis," Journal of the American College of Cardiology, vol. 63, no. 17, pp. 1778-1785, 2014.

[26] K. Mizushige, L. Yao, T. Noma et al., "Alteration in left ventricular diastolic filling and accumulation of myocardial collagen at insulin-resistant prediabetic stage of a type II diabetic rat model," Circulation, vol. 101, no. 8, pp. 899-907, 2000.

[27] D. Aronson, "Cross-linking of glycated collagen in the pathogenesis of arterial and myocardial stiffening of aging and diabetes," Journal of Hypertension, vol. 21, no. 1, pp. 3-12, 2003. 


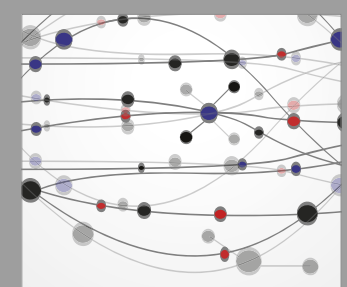

The Scientific World Journal
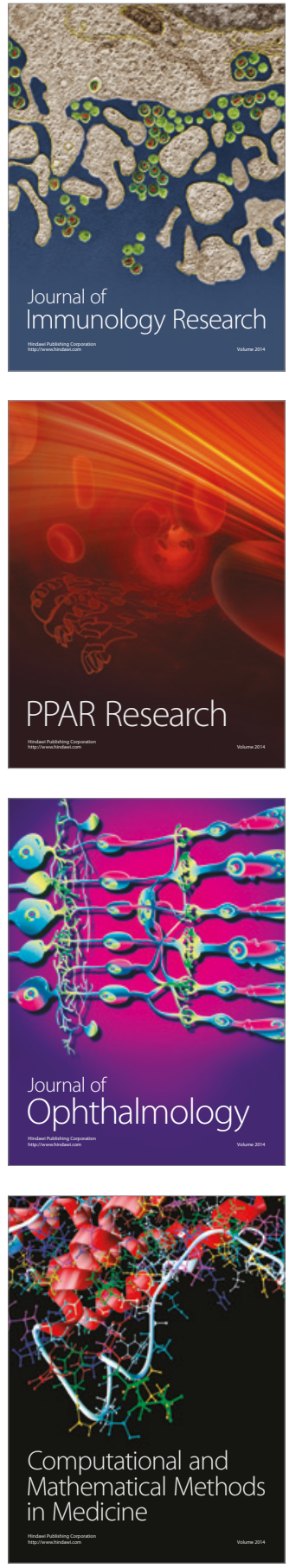

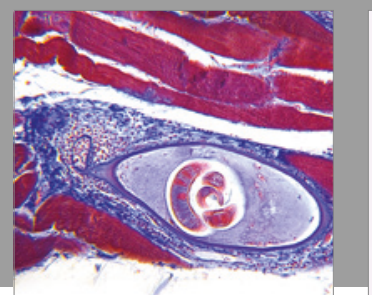

Gastroenterology Research and Practice
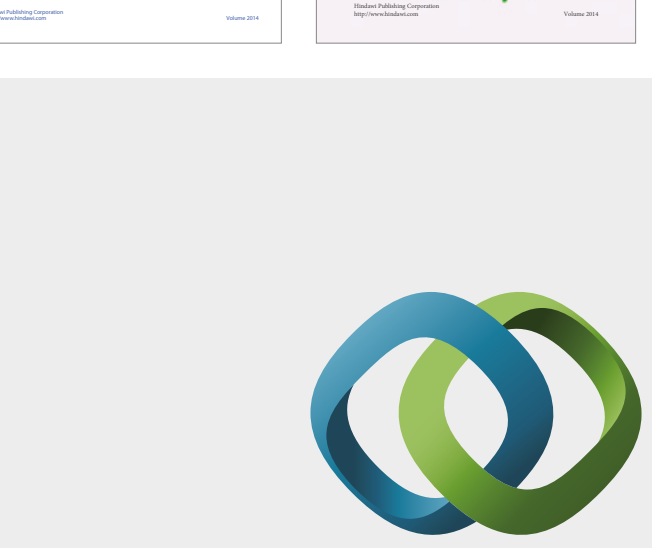

\section{Hindawi}

Submit your manuscripts at

https://www.hindawi.com
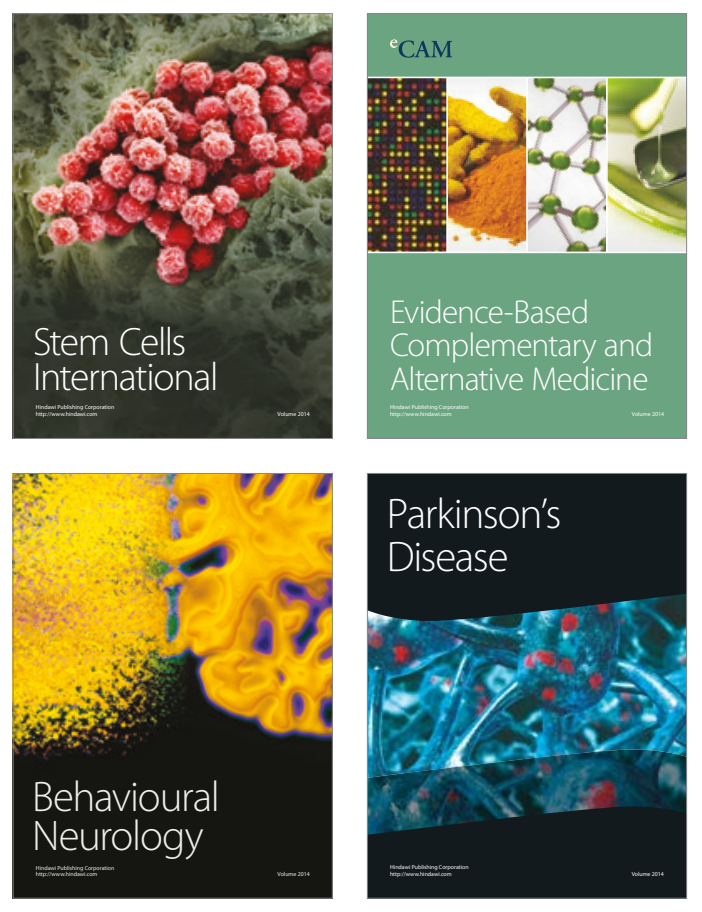
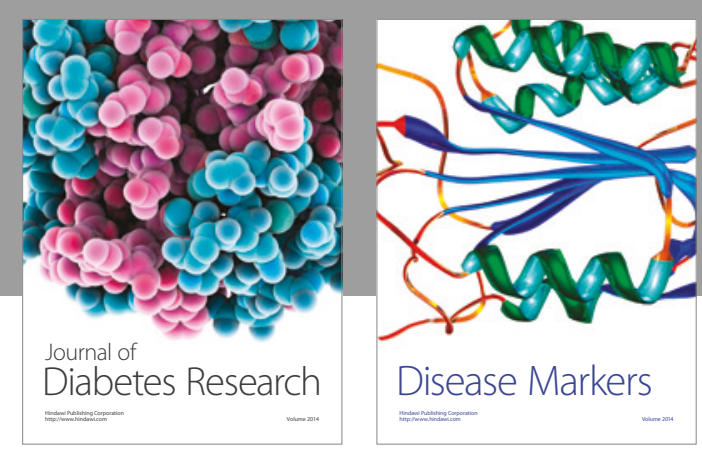

Disease Markers
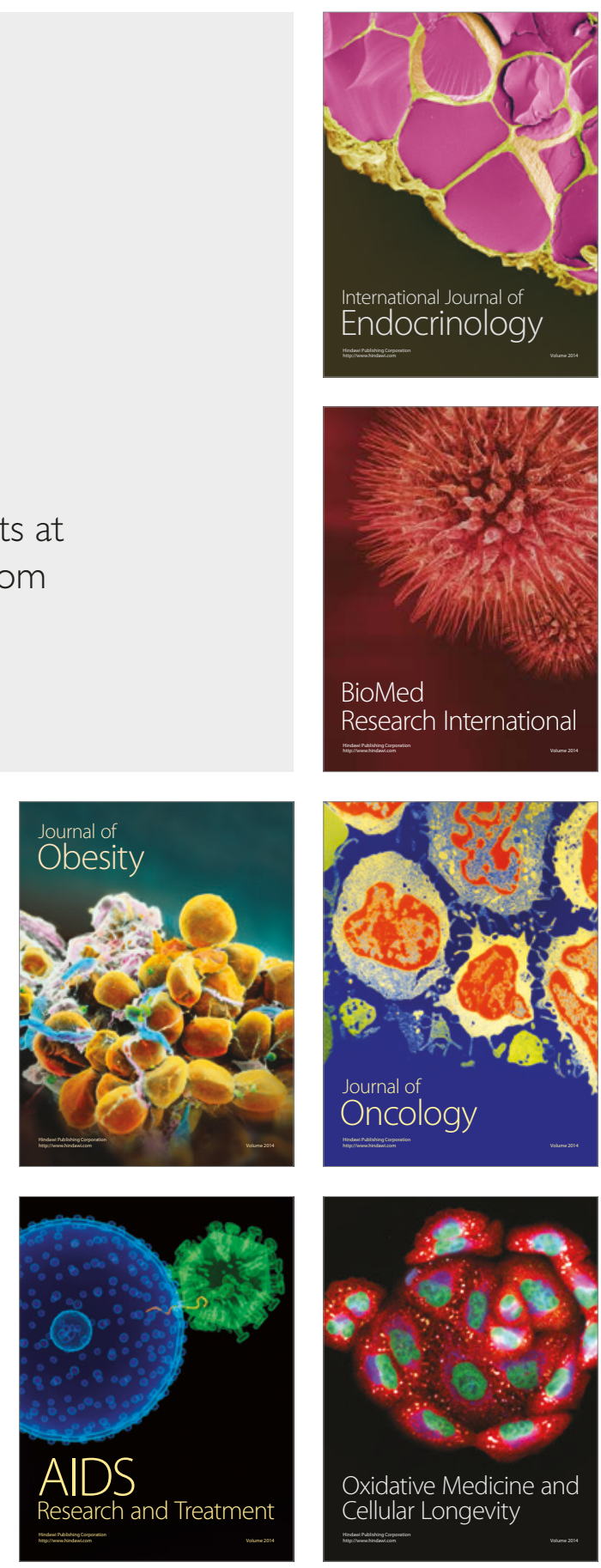